Z. klin. Chem. u. klin. Biochem.

8. Jg., S. $71-76$, Januar 1970

\title{
Elektronenmikroskopische Untersuchungen an einigen Rattenorganen im Mg-Mangelzustand
}

\author{
Von H. J. Merker und Th. GüNTHer \\ Aus dem II. Anatomischen Institut (Dir.: Prof. Dr. W. Schwarz) und dem \\ Pbysiologisch-chemischen Institut (Dir.: Prof. Dr. Dr. E. Schütte) der Freien Universität Berlin
}

(Eingegangen am 29. September 1969)

\begin{abstract}
Von jungen männlichen Albinoratten wurden nach 38tägiger Mg-armer Ernährung Herz, Skelcttmuskel, Nicre und Leber elektronenmikroskopisch untersucht. Am Herz und Skelettmuskel waren das transversale Tubulussystem und der interstitielle Raum erweitert sorvie die submembranösen Cisternen vermehrt und vergrößert. Verstreut über den gesamten Muskelbereich kommen kleine Verkalkungsherde vor. - An der Niere fanden sich eine Schwellung einzelner Zellen, eine Schädigung des Bürstcnsaumes und Zylinderbildungen im Tubuluslumen. Die Verkalkungen sind hier vorwiegend im corticomedullären Gebiet lokalisicrt. - In diesen drei Organen lassen sich Mitochondrienschwellungen, das Platzen einzelner Zellmembranen und Thrombosierungen kleiner Gefäße beobachten. In der Leber können keine signifikanten morphologischen Veränderungen nachgewiesen werden. - Die Ergebnisse werden im Zusammenhang mit biochemischen Ergebnissen diskutiert.
\end{abstract}

\section{Electron microscope studies of some rat organs in a state of $\mathrm{Mg}$-deficiency}

Young, male albino rats wére fed for 38 days on a Mg-deficient diet. The heart, skeletal muscle, kidneys and liver were then studied with the aid of the electron microscope. In heart and skeletal muscle, the transverse tubule system and the interstitial space were increased and the submembranous cisternae were increased in size and number. Small centres of calcification were scattered throughout the muscle. In the kidney individual cells were swollen, the brush border was damaged and the formation of casts was observed in the tubule lumen; the calcification sites were localised chiefly in the cortico-medullary region. Swollen mitochondria, individual burst cell membranes and thrombosed small blood vessels were observed in all three organs. No significant morphological changes could be shown in the liver. The results are discussed in relation to biochemical findings.

In den vorangehenden Mitteilungen $(1,2)$ wurden Veränderungen in der $\mathrm{Na}-, \mathrm{K}$ - und, $\mathrm{Mg}$-Verteilung sowie im Enzymmuster verschiedener Rattenorgane nach Verfütterung einer $\mathrm{Mg}$-armen Nahrung beschrieben. Die Veränderungen waren in der Niere am geringsten und im Skelettmuskel am stärksten ausgeprägt. Sie bestanden im Muskel neben einer geringen Zunahme des Wassergehaltes in einer erheblichen Vermehrung der extrazellulären Flüssigkeit und in einer Abnahme der intrazellulären K-Konzentration. In der Leber nahmen die extrazelluläre Flüssigkeit und die intrazelluläre $\mathrm{K}$ Konzentration ab, während der Wassergehalt und die intrazelluläre $\mathrm{Na}$-Konzentration zunahmen.

Um zu sehen, ob das Ausmaß der Änderungen im Elektrolyt- und Enzymgehalt mit morphologischen Veränderungen korreliert ist, haben wir Teile der gleichen Organe, an denen die anderen Untersuchungen durchgeführt wurden, elektronenmikroskopisch dargestellt. Bisher veröffentlichte licht- und elektronenmikroskopische Ergebnisse lassen sich bei diesem Vergleich nicht heranziehen, da die morphologischen Befunde verschiedener Untersucher große Unterschiede aufweisen. So wurden z. B. bei Mg-arm ernährten Ratten in Muskelzellen keine Veränderungen (3), aber auch Verkalkung und Schwellung der Sarcosomen, sowie Fragmentation der Myofibrillen $(4,5,6)$ gefunden. In der Niere wurden starke oder geringe Veränderungen mit Verkalkungen bzw. geringe Veränderungen ohne Verkalkungen beschrieben (7), wobei unter Veränderungen Mitochon- drienschwellung, vermehrte Einschlußbildung und Schädigung des Bürstensaumes zu verstehen sind.

\section{Material und Methoden}

Die Vorbehandlung der Versuchstiere wurde bereits ausführlich beschrieben (1). Die Organe (Leber, Niere, Herzmuskel, Skelettmuskel) wurden sofort nach Entnahme mit einer 1proz. $\mathrm{OsO}_{4}$-Lösung in 0,1M Phosphatpuffer $\mathrm{pH} 7,2$ für 2 Stdn. fixiert, danach in der Acetonreihe entwässert und in Micropal ( $\mathrm{F}_{2}$. Ferak, Berlin) eingebettet. Schnitte: LKB-Mikrotome; Nachkontrastierung: Uranylacetat/Bleicitrat; Aufnahmen: Siemens-Elmiskope I und Ia.

Zur lichtmikroskopischen Kontrolle und zur Beobachtung größerer Flächen wurden $1 \mu \mathrm{m}$ dicke Schnitte hergestellt, in 50proz. Giemsa-Stammlösung (wäßr. mit Boraxzusatz) gefärbt und im Lichtmikroskop beurteilt.

\section{Befunde}

Herzmuskel

In elektronenmikroskopischen Übersichtsaufnahmen lassen sich in annähernd regelmäßigen Abständen runde Aufhellungszonen zwischen den Myofilamentbündeln (Myofibrillen) nachweisen. Bei höherer Auflösung wird deutlich, daß es sich um membranbegrenzte Hohlräume handelt. In ihrer engen Nachbarschaft liegen noch andere Hohlräume, die jedoch nicht erweitert sind und eine tubuläre Form haben. Die erweiterten Abschnitte sind deshalb dem T-System (transversales S.) des sarcoplasmatischen Retikulums zuzuordnen, die nicht erweiterten dagegen dem longitudinalen System. Für diese Deutung spricht auch das Vorkommen von basalmembranähn- 
lichem Material im T-System, das als tiefe Einsenkung der Zellmembran in das Cytoplasma zu verstehen ist. Die Basalmembran macht dabei diese Einstülpung auf weiten Strecken mit. Die fast regelmäßige Anordnung der tubulären Hohlräume des T-Systems und damit der Erweiterungen wird durch ihre enge räumliche Beziehung zum Z-Streifen verständlich (Abb. 1).

In lichtmikroskopischen und in nur gering vergrößerten elektronenmikroskopischen Aufnahmen kommt noch eine Erweiterung des interstitiellen Raumes zum Ausdruck. In den optisch leeren Räumen zwischen den kleinen Fibrillenbündeln des Bindegewebes und den abgrenzenden Basalmembranen der Muskelzellen und Kapillaren lassen sich auch bei hoher Vergrößerung keine Strukturen darstellen.

Als weiterer Befund ist das veränderte Verhalten des Raumes dicht unter der Zellmembran zu erwähnen. Hier sind im Kontrollmuskel nur wenige Vesikel von 500-1000 § Durchmesser nachzuweisen. In den Herzmuskelzellen der Mg-Mangel-Tiere dagegen kommen im Raum zwischen Zellmembran und dem peripheren Myofilamentbündel zahlreiche runde und langgestreckte Cisternen vor, die eine Breite von $0,3 \mu \mathrm{m}$ und eine Länge von $0,6 \mu \mathrm{m}$ erreichen und keinen Inhalt haben. Die Mitochondrien sind in den meisten Zellen leicht geschwollen. Diese Schwellung äußert sich in einer Abrundung der Mitochondrien, die sonst überwiegend längsoval sind. Ihre Matrix ist verschwunden und die Cristae heben sich dadurch klar hervor. Teilweise hat sich auch der Abstand zwischen den Cristae vergrößert (Abb. 1). An einzelnen Stellen ist die Zellmembran geplatzt. Hier strömen Cytoplasmabestandteile, besonders die oft peripher angehäuften Mitochondrien aus. Sie sind dann in dem erweiterten interzellulären Raum relativ gut erhalten' nachzuweisen. Die betroffenen Muskelzellen zeigen keine weiteren Veränderungen, etwa im Sinne einer Schwellung. Wahrscheinlich wirken die Myofibrillen stabilisierend auf das Zellgefüge (Abb. 1). Darüber hinaus sieht man zahlreiche Kapillaren, die mit dichtgepackten Thrombocyten ausgefüllt sind. Zwischen ihnen läßt sich ein fibrilläres elektronendichtes Material darstellen, das bei höherer Vergrößerung eine feine Querstreifung mit einer Periodenlänge von $200 \AA$ zeigt und deshalb als Fibrin identifiziert werden kann. Derartige Thrombocytenausmauerungen kommen auch in den Kapillarbezirken der anderen Organe vor.

Im Herzmuskel finden sich außerdem intra- und interzellulär gelegene Kalkdrusen. Sie bestehen aus lanzettförmigen Nadeln, die bis zu $0,1 \mu \mathrm{m}$ lang und $300-500 \AA$ breit sind. Diese Kalknadeln sind häufig zu einem dichten Zentrum zusammengelagert, um das eine optisch leere Zone liegt. An der Peripherie schließt ein Ring mit häufig radiär gestellten Nadeln den Einschluß ab. Die Drusen sind vorwiegend rund, es können auch mehrere zu traubenförmigen Gebilden zusammenfließen. In ausgedehnten Verkalkungsbezirken ist ein Aufbau aus Einzeldrusen und eine ringförmige Schichtung der Nadeln nicht mehr nachweisbar. Die Kalkniederschläge liegen manchmal im Matrixraum zwischen den Cristae
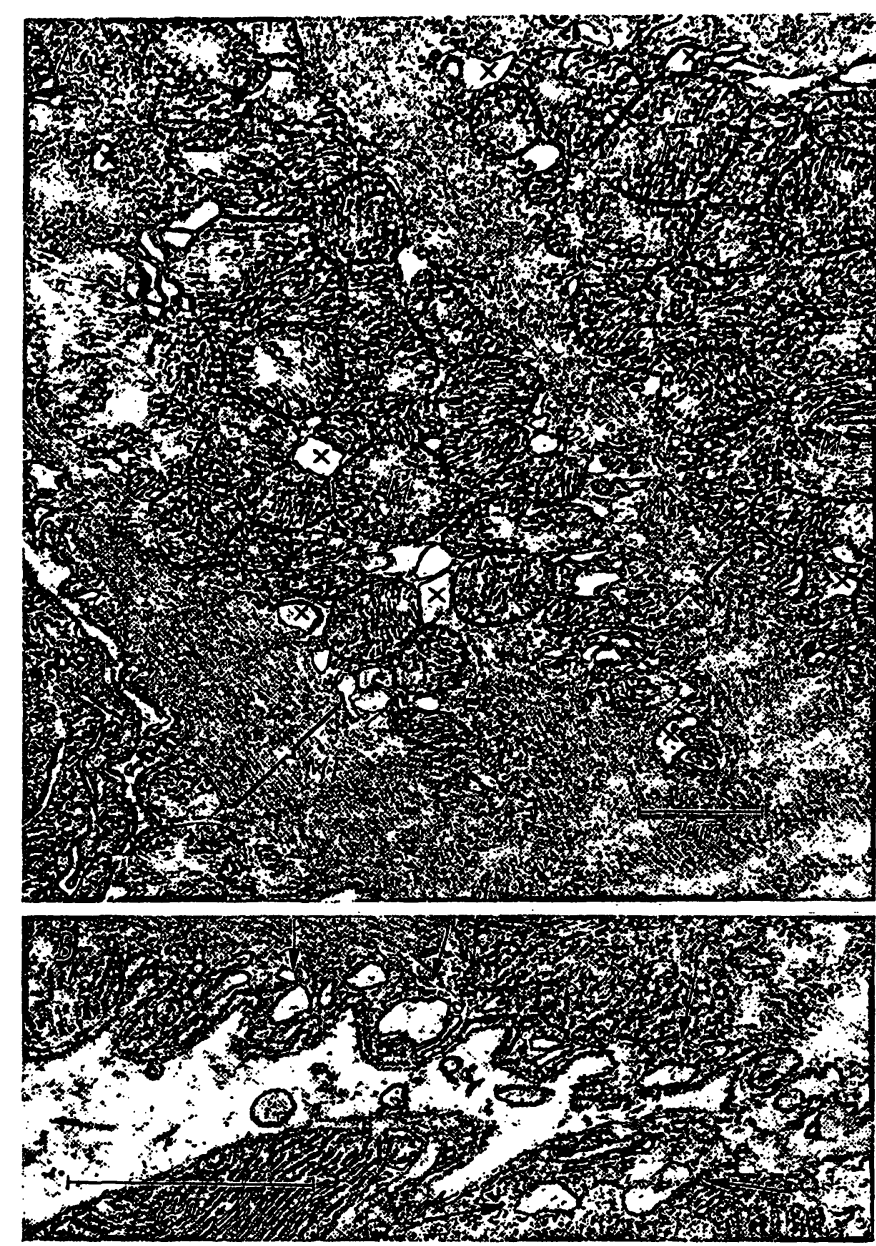

Abb. 1

A. Herzmuskel. Leichte Mitochondrienschwellung (M), Erweiterung des T-Systems $(X)$. MF $=$ Myofibrillen
Vergrößerung $1: 12000$

B. Herzmuskel. Erweiterung und Vermehrung der submembranösen Cisternen ( $\downarrow)$

der Mitochondrien. Ihr bevorzugter Entstehungsort scheint aber das sog. Grundcytoplasma in der Nachbarschaft der Organellen und die Grundsubstanz des Bindegewebes zwischen den Fibrillenbündeln zu sein. In der Zelle selbst liegen die Kalkniederschläge entweder frei oder durch eine Membran vom übrigen Cytoplasma abgegrenzt.

\section{Skelettmuskel}

Hier kommen die gleichen, wie beim Herzmuskel geschilderten Veränderungen vor: Erweiterungen des interstitiellen Raumes und des sarcoplasmatischen Retikulums (T-System), Vermehrung und Erweiterung der submembranösen Cisternen, leichte Mitochondrienschwellung, vereinzeltes Platzen der Zellmembranen mit Ausströmen von Cytoplasmabestandteilen, Thrombocytenaggregationen und Fibrinausfällungen. Kalkeinlagerungen konnten wir im Skelettmuskel nur selten und wenig ausgedehnt nachweisen (Abb. 2, 3).

In der Niere finden sich vereinzelte Mitochondrienschwellungen; die im elektronenmikroskopischen Bild durch Abrundung, Vergrößerung, Matrix- und Cristaeverlust deutlich werden. Einzelne Zellen können stärker betroffen sein als andere. In einer Zelle liegt häufig ein geschwollenes Mitochondrium neben vielen unveränder- 


\section{Präzision Richtigkeit Identifizierung}

\section{in der klinischen Chemie durch Automatisierung}

von der Probendosierung bis zum Ausdrucken von Probennummer und Analysenergebnis.

Der ANALYSENAUTOMAT C4 mißt photometrisch Glucose, Bilirubin, Harnstoff-N, Kreatinin, Cholesterin, Total-Protein, GOT, GPT, Phosphatase-S; Phosphatase alk., Harnsäure, Chlorid, Phosphor, SerumEisen, CPK, LDH, Albumin, sowie mit dem Flammenphotometer auch Kalium und Natrium.

120 Analysen pro Stunde. Bis zu vier Bestimmungen aus einer Probe. Automatischer Abgleich auf Reagenzien- und Serumleerwert.

Probenidentifizierung und Auswertung auch auf Blattschreiber oder mit Datenverarbeitungssystem.

Neben dem Vierkanal-Analysenautomat $C 4$ liefern wir für die klinische Chemie auch UV-SPEKTROPHOTOMETER und SPEKTROPHOTOFLUORIMETER SOWIe ATOM-ABSORPTIONS-SPEKTROPHOTOMETER für Routinemessungen von $\mathrm{Ca}, \mathrm{Mg}, \mathrm{Fe}, \mathrm{Cu}, \mathrm{Zn}$, u. a. in Körperflüssigkeiten.

Atom-Absorptions-Spektrophotometer mit der neuartigen Boot-Technik zur Direktbestimmung von $\mathrm{Pb}, \mathrm{Tl}, \mathrm{As}, \mathrm{Hg}$ u. a. in Urin oder Blut.

BODENSEEWERK PERKIN-ELMER \& CO GMBH

Vertrieb Klinische Chemie, 6 Frankfurt, Schöne Aussicht 16

Tel. (0611) 283487 , Telex 0411827
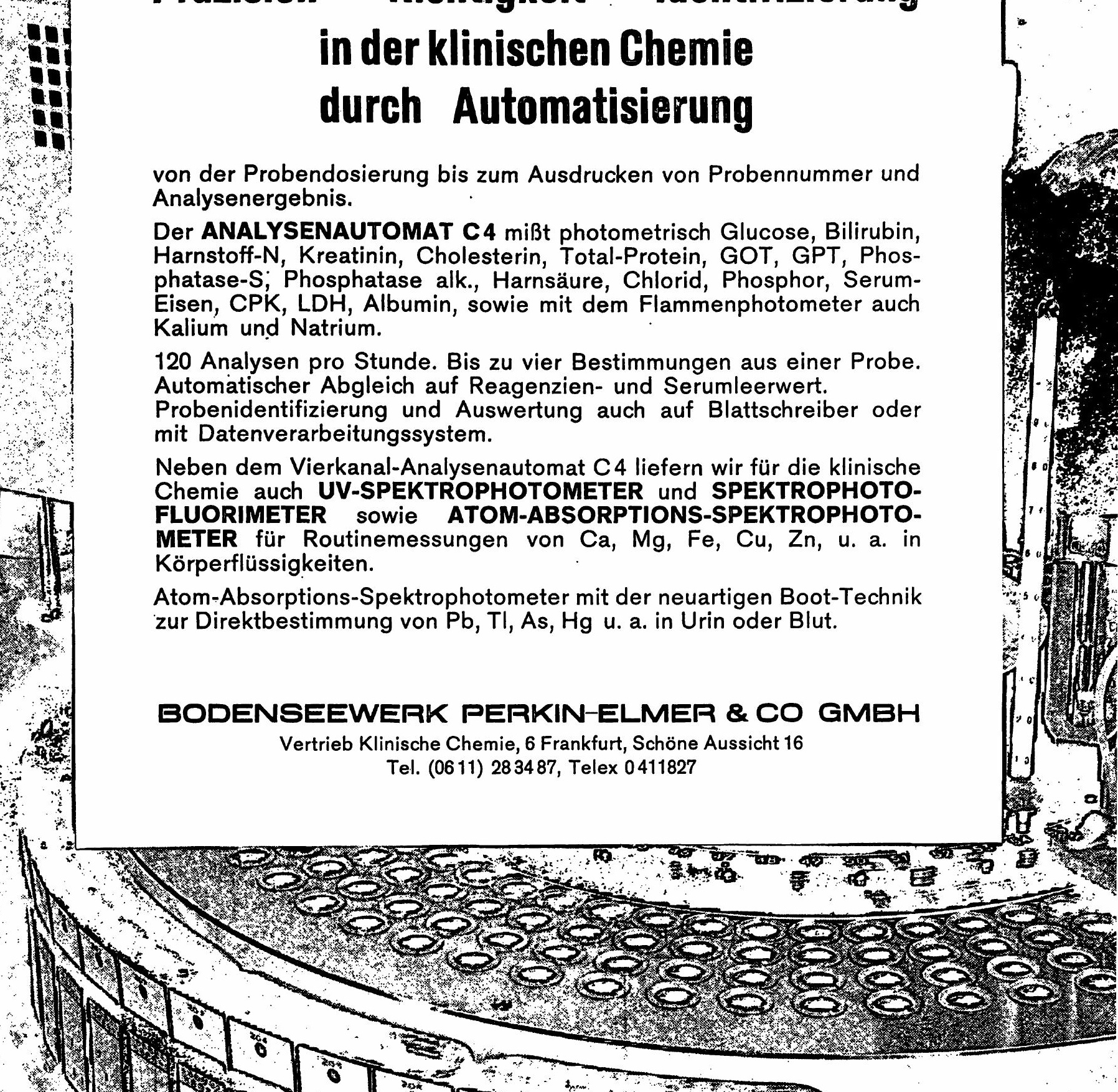


\section{Hoppe-Seyler's Zeitschrift für Physiologische Chemie}

Nachdruck Band 1-282 (1877-1945) züzüglich 8 Registerbände

Subskriptionspreis broschierte komplette Serie DM 15 600,-

Zur Komplettierung unvollständiger Serien werden nachstehend genannte Einzelbände zur Subskription gestellt. Subskriptionspreis broschiert je DM 70,-:

$\begin{array}{rrrll}2 & 17 & 75 & 177 & 269 \\ 6 & 18 & 81 & 182 & 270 \\ 8 & 20 & 82 & 203 & 271 \\ 10 & 23 & 92 & 211 & 275 \\ 11 & 31 & 132 & 212 & 276 \\ 12 & 33 & 141 & 218 & 280 \\ 13 & 34 & 175 & 219 & \text { Reg. Bd. } \\ 15 & 69 & 176 & 235 & 226-250\end{array}$

Der Nachdruck kann erst vorgenommen werden, wenn eine ausreichende Anzabl von Subskriptionen vorliegt. Alle hier nicht aufgeführten Bände der Serie sind noch in geringer Anzahl lieferbar. Der Ladenpreis für den broschierten Einzelband beträgt DM 85,-

Walter de Gruyter \& Co · Berlin 


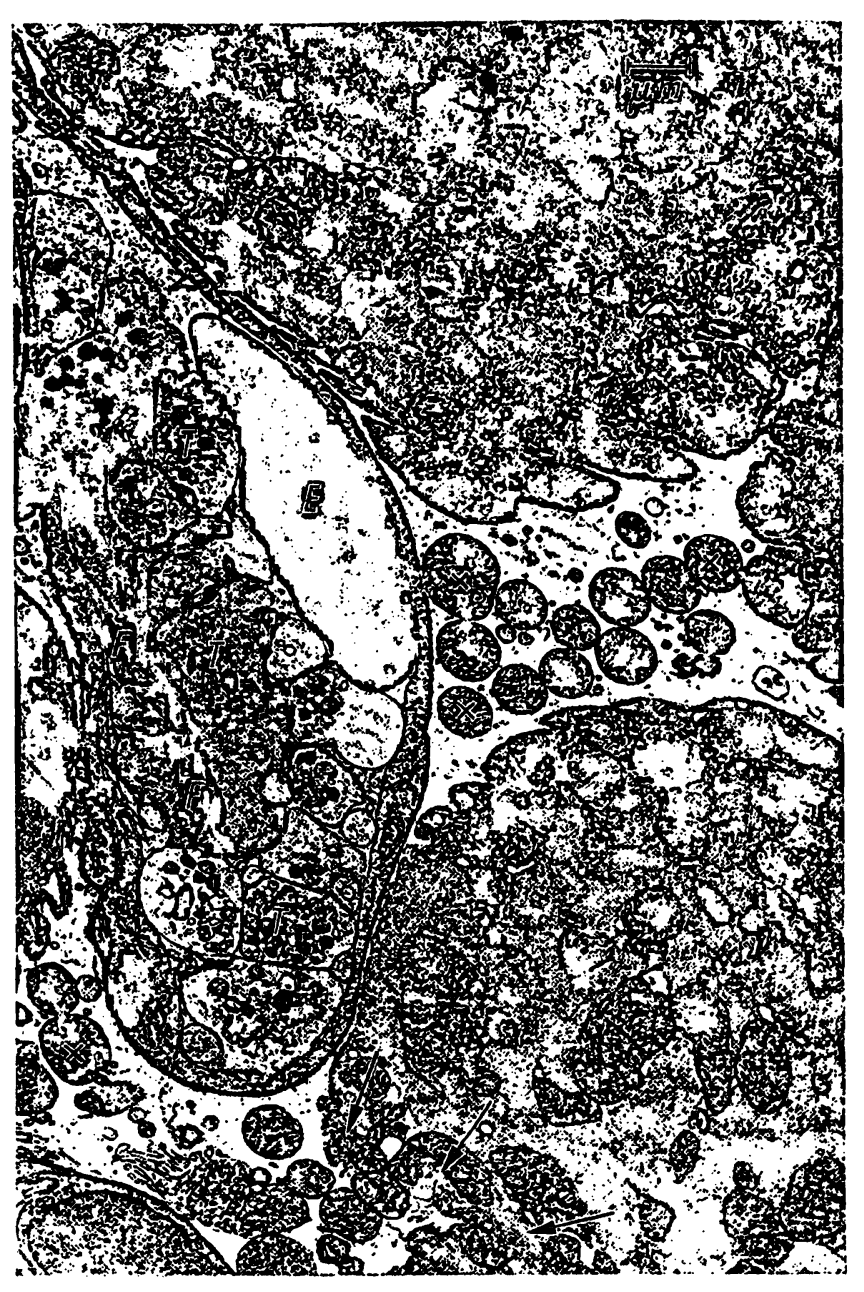

Abb. 2

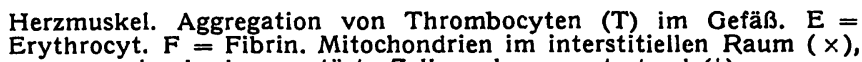

durch eine zerstörte Zellmembran austretend $(\downarrow)$
Vergrößerung 1:5000

ten. Die Zahl der elektronendichten Einschlüsse und der Vakuolen im proximalen Abschnitt des Nephrons ist erhöht. An der Grenze zwischen Cortex und Medulla liegen vermehrt Kalkablagerungen, die den Drusen in Muskelzellen entsprechen. Der Bürstenbesatz des proximalen Tubulusabschnittes zeigt stellenweise Schädigungen. Hier blähen sich einzelne Mikrovilli auf und ragen dann blasenförmig in das Lumen. Auch apikale Abschnitte der Tubuluszellen können sich weit in das Lumen hinein vorwölben. An einzelnen Stellen ist die apikale Zellmembran gerissen und es strömen Zellorganellen in das Lumen aus. Auch andere granuläre Substanzen lassen sich hier nachweisen, so daß man von Zylinderbildung sprechen kann (Abb. 4, 5, 6). In der Leber lassen sich bei den Mg-Mangel-Ratten elektronenmikroskopisch keine deutlichen Abweichungen gegenüber der Norm nachweisen (Abb. 7).

\section{Diskussion}

Die im Muskel vọn Mg-arm ernährten Ratten gefundene Vermehrung des $\mathrm{Na}-$ Gehaltes und der extrazellulären Flüssigkeit (1) entspricht der Vergrößerung des interstitiellen Raumes und der Erweiterung des T-Systems, das mit der extrazellulären Flüssigkeit in Verbindung steht (s. u.). Wahrscheinlich sind an der Vermehrung

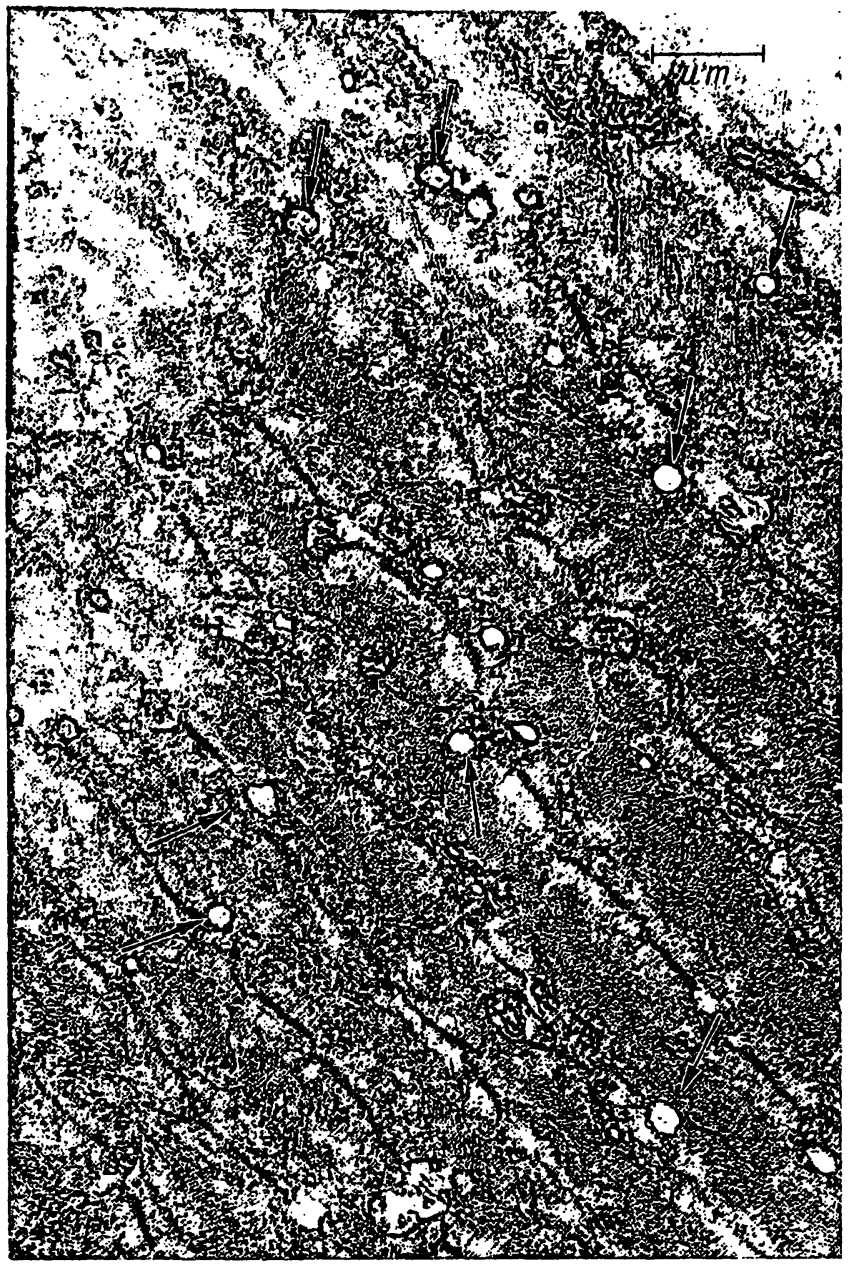

Abb. 3

Skelettmuskel fast im Querschnitt. Leichte Mitochondrienschwellung (M). Erweiterung des T-Systems $(\downarrow)$
Vergrößerung 1:11000

der extrazellulären Flüssigkeit auch die unter der Membran gelegenen Cisternen beteiligt, die im normalen Muskel nur vereinzelt sichtbar sind. Sie könnten durch ein Zusammenfließen von pinocytotischen Bläschen, die immer in der Zellmembran der Muskelzellen zu beobachten sind, entstehen. Als Voraussetzung müßten vermehrte Vesikulationsvorgänge angenommen werden. Sie könnten aber auch Hohlräume des longitudinalen endoplasmatischen Retikulums sein, die dann in gröBerer Zahl unter der Zellmembran liegen und, wie das transversale System, etwas aufgebläht wären. $\mathrm{Da}$ das longitudinale und transversale Tubulussystem an einzelnen Stellen miteinander verbunden ist (8), könnte der vergrößerte Innenraum dieser Cisternen in beiden Fällen extrazelluläre Flüssigkeit enthalten.

Das transversale Tubulussystem des endoplasmatischen Reticulums steht im Muskel mit der extrazellulären Flüssigkeit in offener Verbindung, nicht dagegen das endoplasmatische Reticulum anderer Zellen, bei dem zwischen endoplasmatischem Reticulum und extrazellulärer Flüssigkeit ein steter Vesikelfuß von der Zellmembran zum endoplasmatischen Reticulum und umgekehrt existiert (9). Bei einer Steigerung dieser Vorgänge kann es praktisch zur offenen Verbindung zwischen Extrazellulär-Raum und endoplasmatischem Reti- 


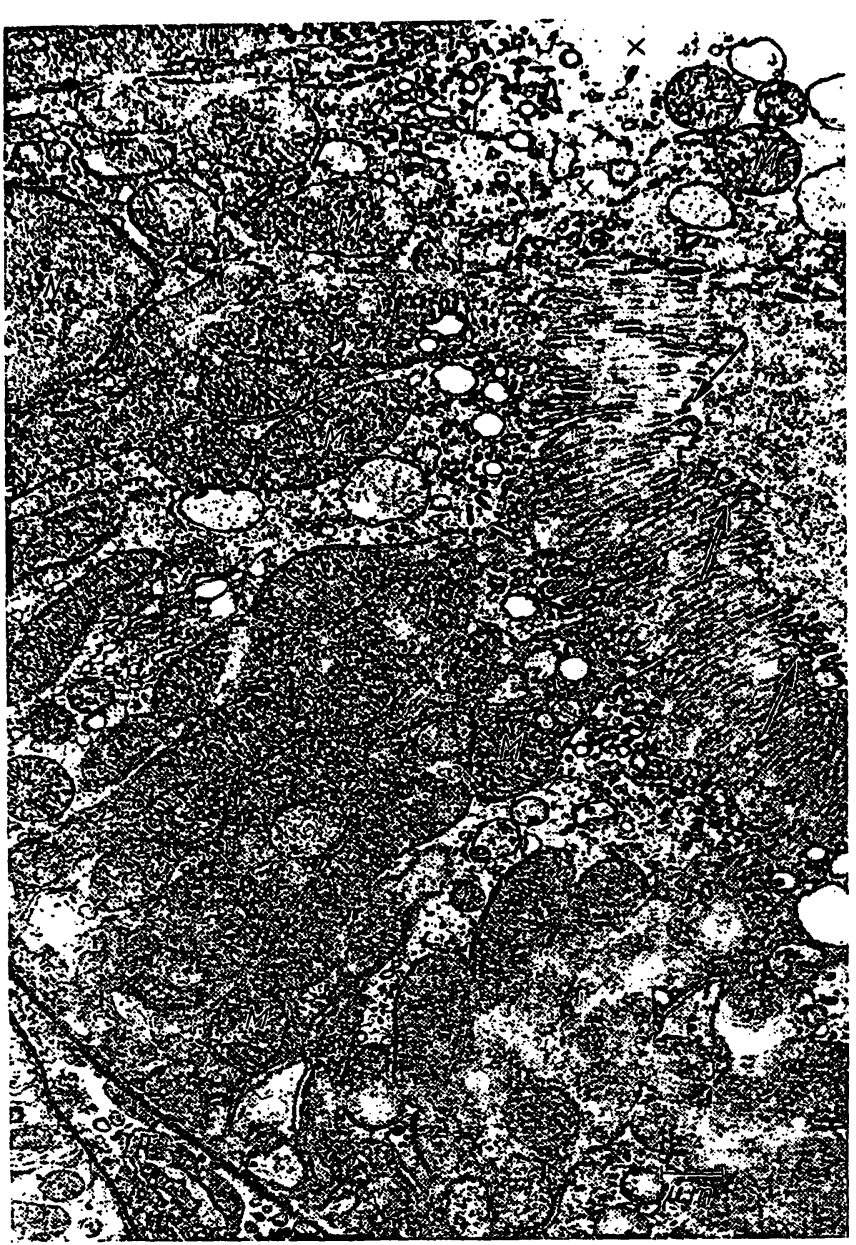

Abb. 4

Proximaler Tubulusabschnitt (Hauptstück). Granuläre Substanz im Lumen (L), kolbenförmige Auftreibung der Mikrovilli $(\downarrow)$, leichte
Mitochondrienschwellung (M), Aufhellung des Grundcytoplasmas $(X)$. $\mathrm{N}=$ Nucleus, $\mathrm{IR}=$ Interstitieller Raum

Vergrößer ung 1:5000

culum kommen; allerdings ist die Verbindung immer noch zellulär kontrolliert. Das T-System des Muskels sollte deswegen und wegen der morphologischen Ähnlichkeit seiner Membran mit der Zellmembran (deutliche „unitmembrane" und Basalmembranbegleitung) nicht als Sonderform des endoplasmatischen Reticulums bezeichnet werden. Mit der Möglichkeit seiner Erweiterung und Verkleinerung erinnert es eher an das basale Labyrinth der Niere oder an den Interzellularraum einschichtiger Epithelverbände (10,11). Unsere Befunde sprechen also für die Möglichkeit einer Vermehrung der extrazellulären Flüssigkeit durch Erweiterung intrazellulär gelegener Hohlräume (bei nur geringer Erweiterung des interstitiellen Raumes). Ähnliche Vergrößerungen des Extrazellulär-Raumes und des endoplasmatischen Reticulums fanden wir früher an der Rattenleber nach Cortisongaben (12) und an Skelettmuskelzellen der Ratte nach Behandlung mit Methylthiouracil (13).

Im Herzmuskel von Mg-arm ernährten Ratten waren die morphologischen Veränderungen stärker als im Skelettmuskel. Gegenüber den anderen quergestreiften Muskeln enthält die Herzmuskelzelle mehr und größere Mitochondrien mit mehr Matrix und Cristae. Das T- und L-System des Herzmuskels ist deutlicher ausgeprägt

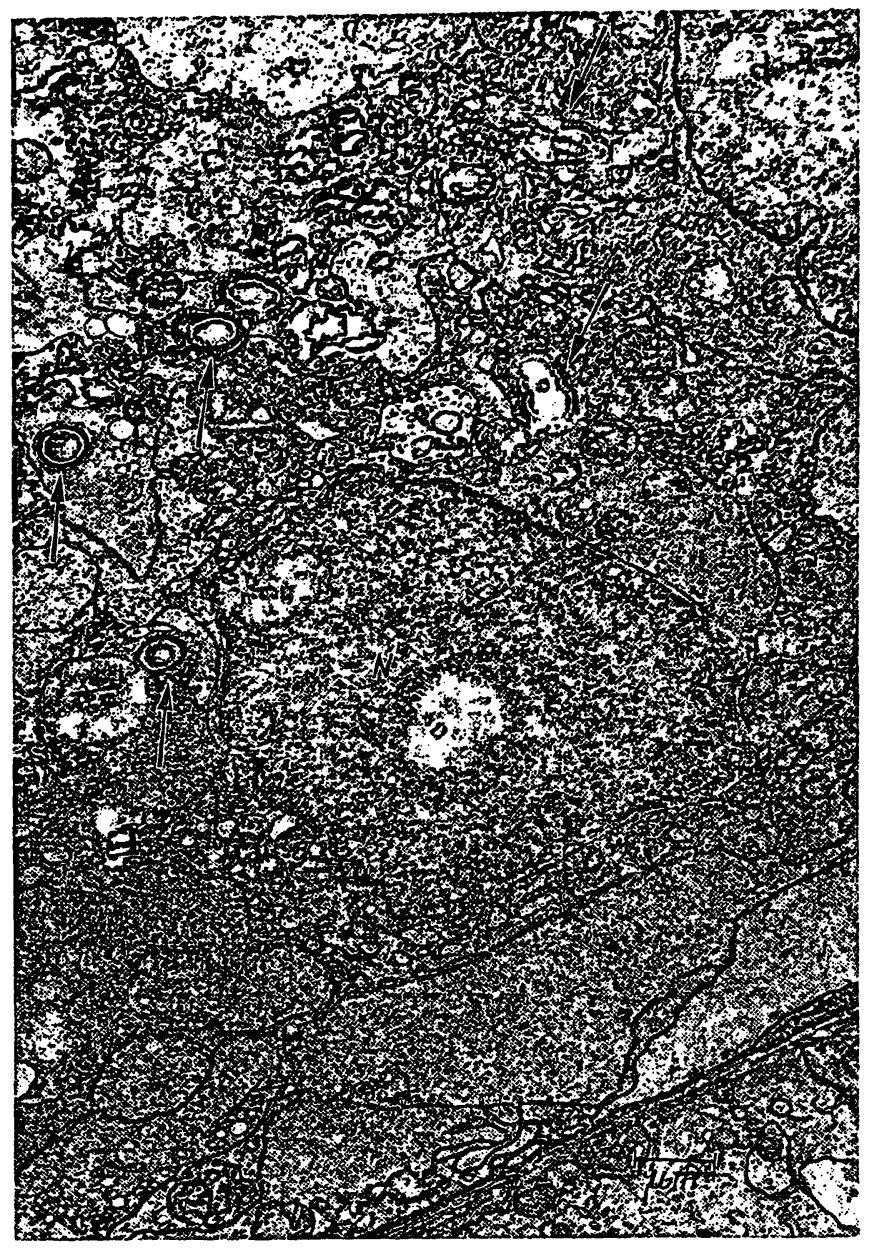

Abb. 5

Geschädigte Zelle aus der $N$ Niere (Hauptstück) mit Kalkdrusen $(\downarrow)$. Vergrößerung 1:7000

und geordnet und deshalb besser darzustellen. Die Breite des interzellulären Raumes schwankt hier nicht in so weiten Bereichen. Eine Veränderung dieser Strukturen wird deshalb im Herzmuskel deutlicher sichtbar als in den anderen Muskelverbänden. Die Zunahme des Naund die Abnahme des $\mathrm{K}$ - und $\mathrm{Mg}$-Gehaltes waren im Herzmuskel jedoch geringer als im Skelettmuskel (1). Ein exakter quantitativer Vergleich morphologisch und chemisch ermittelter Veränderungen zwischen Herz- und Skelettmuskel ist aber nicht möglich, da im Herzmuskel die Größe der extrazellulären Flüssigkeit nicht ermittelt wurde.

An der Niere wurden keine signifikanten Änderungen im Na-, K- und im Gehalt der getesteten Enzyme festgestellt $(1,2)$, obgleich morphologische Veränderungen an den Tubuluszellen mit extra- und intrazellulären Verkalkungen besonders im corticomedullären Bereich zu sehen waren. Hier ergibt sich die Frage, ob die sichtbaren Veränderungen primär oder sekundär sind. Das Platzen der Zellmembran und die Neigung der Niere, besonders im corticomedullären Grenzgebiet $\mathrm{zu}$ verkalken, stört den Abfluß im Nephron und fördert die Zylinderbildung. Zelltrümmer und Zylinder werden aber die Morphologie des gesamten Nephrons beeinflussen. Auch die lichtmikroskopisch sichtbare und von 


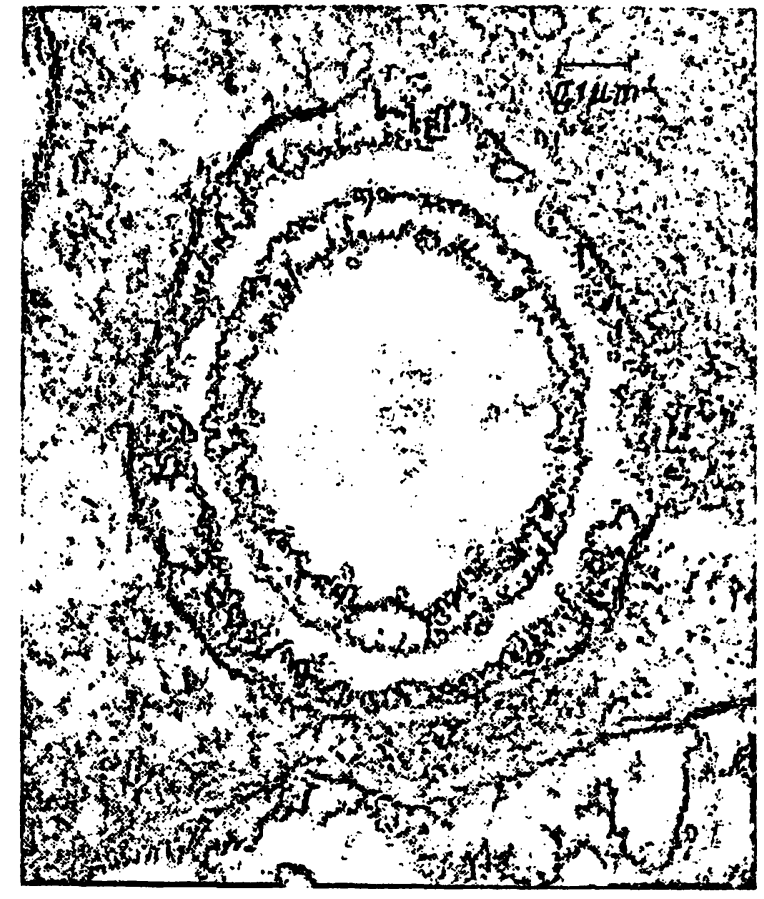

Abb. 6

Intrazelluläre (I) und extrazelluläre (E) Kalkablagerungen in der Niere Vergrößerung 1:70000

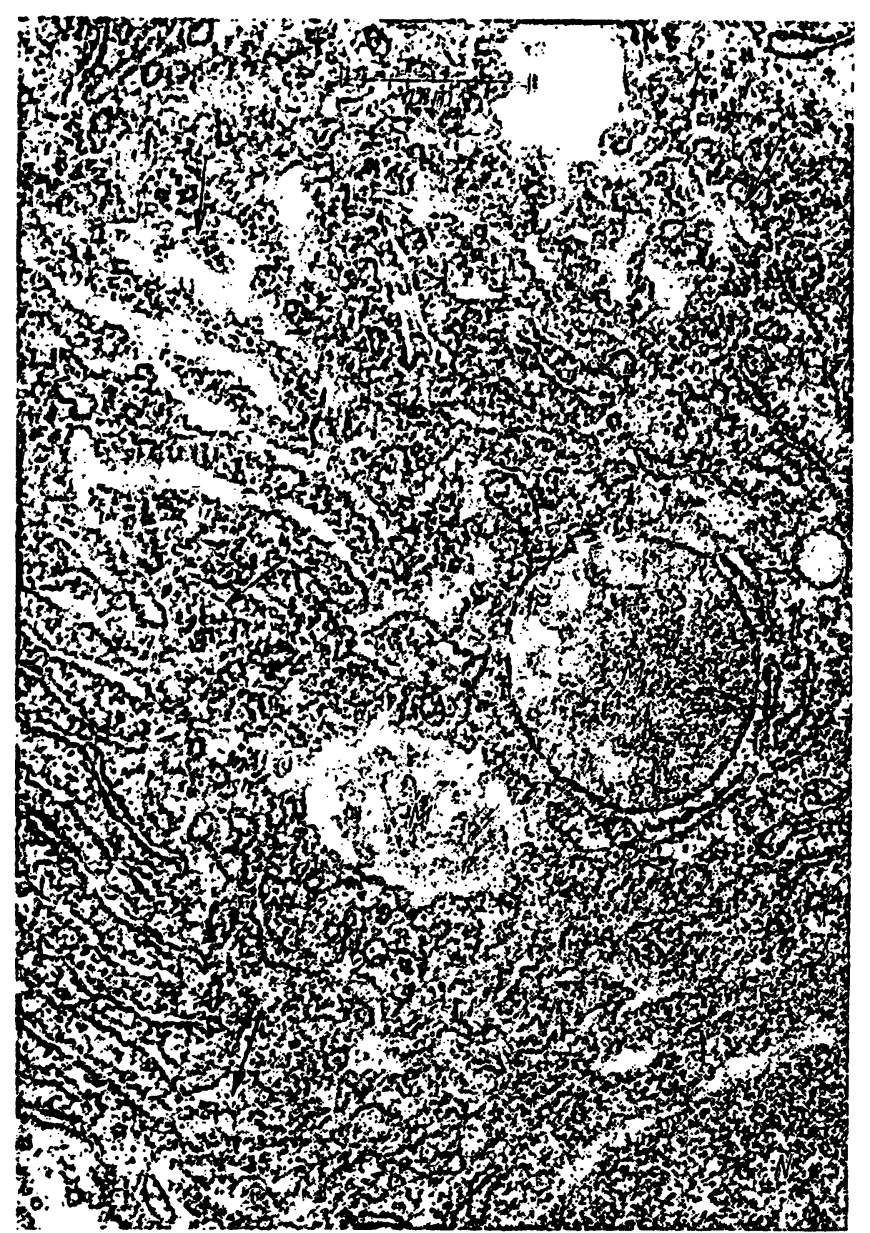

Abb. 7

Leber. Typische Ribosomen in Rosettenform (Polysomen). $M=$ Mitochondrien, $N=$ Nucleus
Vergrößerung $1: 18000$ anderen Untersuchern (7) gefundene Mesenchymreaktion mit einer Vielzahl kleinster Entzündungsherde und Narbenbildung ist als Sekundärreaktion auf den Untergang einzelner Zellen und die dissemierten Kalkablagerungen $z u$ deuten.

In der Leber war die extrazelluläre Flüssigkeit und der aktive Na-K-Transport vermindert (1) und die Aktivität einiger Enzyme erhöht (2). Es waren jedoch keine deutlichen morphologischen Änderungen an den Zellen und Zellorganellen eingetreten. Dies zeigt, daß eine Korrelation zwischen chemischen und morphologischen Befunden nicht in allen Fällen möglich ist.

An allen Zellen der $\mathrm{Mg}$-arm ernährten Tiere waren im Gegensatz zu Mg-arm gewachsenen Bakterienzellen keine signifikanten qualitativen und quantitativen Änderungen der Ribosomen zu sehen. Das liegt wahrscheinlich an der nur geringen Abnahme des $\mathrm{Mg}$-Gehaltes, die bei tierischen Zellen durch $\mathrm{Mg}$-arme Nahrung eintritt. Eine Veränderung dieser $\mathrm{Mg}$-abhängigen intrazellulären Strukturen erfolgt deshalb nicht. Anders verhält es sich offenbar mit den Zellmembranen, deren Stabilität - wahrscheinlich durch die starke Abnahme der extrazellulären $\mathrm{Mg}$-Konzentration - geringer wird, so daß Zellen platzen und ihre Zellorganellen in den interstitiellen Raum austreten. Solche Bilder können auch als Artefakte erklärt werden, die während der Präparation entstehen. Unter den gleichen methodischen Bedingungen haben wir jedoch niemals ein Platzen der Zellmembran mit Ausfließen des Zellinhaltes gesehen. Auch die Aktivitătszunahme einiger Enzyme im Serum (2) spricht für eine Labilisierung der Zellmembran mit einem Freiwerden des Zellinhaltes.

Zur Erklärung der Mitochondrienschwellung, die in allen untersuchten Organen mit Ausnahme der Leber gefunden wurde, lassen sich einmal die Konzentrationsänderungen der intrazellulären Alkali-Ionen heranziehen. Eine Verminderung der K-Konzentration kann bei isolierten Mitochondrien eine Schwellung bewirken (14), die durch eine gleichstarke Zunahme der NaKonzentration nur $z$. T. verhindert wird (14).

Außerdem nimmt bei $\mathrm{Mg}$-Mangeltieren der $\mathrm{Ca}-\mathrm{Gehalt}$ besonders in Niere und Herz, in der Leber aber nur geringfügig zu $(15,16)$. Aus in vitro-Versuchen ist bekannt, $\mathrm{da} B \mathrm{Mg}$ Mitochondrienschwellung verhindert, während $\mathrm{Ca}$ diese induzieren kann $(17,18)$. Damit ließe sich das Fehlen signifikanter morphologischer Veränderungen in der Leber erklären.

$\mathrm{Zu}$ diskutieren ist weiterhin, warum in manchen Zellen nur einzelne Mitochondrien bzw. Sarcosomen schwellen. Es wäre möglich, daß die Änderungen des Na-, K-, Mgund $\mathrm{Ca}-$ Gehaltes im Cytoplasma nicht gleichmäßig eingetreten sind. Es könnten jedoch auch Struktur- und 
Funktionsunterschiede zwischen den Mitochondrien innerhalb einer Zelle bestehen (19).

Die Zahl der Mitochondrien erscheint in unseren Aufnahmen nicht vermindert. Mrshra (20) hatte dagegen beim Auszählen der Mitochondrien nach Homogenisie- ren der Organe von $\mathrm{Mg}$-arm ernährten Tieren eine $\mathrm{Ab}$ nahme der Mitochondrienzahl gefunden. Es fragt sich, ob ein Teil der geschwollenen Mitochondrien bei der Präparation zerstört wurde.

\section{Literatur}

1. GÜNTHER, TH., diese Z. 8, 65 (1970). - 2. GüNTHER, TH., diese Z. 8, 69 (1970). - 3. Goldsmith, L. A., J. Nutrit. 93, 87 (1967). 4. Heggtveit, Life Sci. 4, 69 (1965). - 5. Lowenhaupt, E.,.M. P. Schulman und D. M. Greenberg, Arch. path. Anat. 49, 427 (1950). - 6. Mishra, R. K., Rev. Canad. Biol. 19, 122 (1960). 7. Sabour, M. S., S. Hanna und M. K. McDonnald, Quart. J. Exper. Physiol. 49, 314 (1964). - 8. Girardier, L., W. G. ForssmanN, A. Hyde und A. Matter, Anat. Anz. 121, Erg.-Heft 62, 57 (1968). - 9. Porter, K. R., in: Biological Structure and Function, Academic Press London-New York 127 (1961). - 10. Herzer, R., H. J. Merker und F. J. Haberich, Zschr. exper. Med. 150, 239 (1969). - 11. Diamond, J. M. und E. M. Wright, Annual Rev. Physiol. 31, 581 (1969). - 12. GoeCKE, C., Th. GüNther, H. J.
Dulce und H. J. MÊrKer, Naunyn-S'chmiedebergs Arch. exp. Pathol. Pharmakol. 256, 79 (1967). - 13. GüNrrier, TH., C. Goecke und J. Wolfp, Naunyn-Schmiedebergs Arch. exp. Pathol. Pharmakol. 258, 280 (1967). - 14. Blondin, G. A., W. J. VAIL und D. E. GreEN, Arch. Biochem. Biophysics 129, 158 (1969). - 15. McInTYRe, I. und D. Davidson, Biochem. J. 70, 456 (1958). - 16. Martindale, L. und F. W. Heaton, Biochem. J. 92, 119 (1964). - 17. RaAfLaUb, J., Helv. physiol. Pharmak. Acta 11, 142 (1953), 11, 153 (1953). - 18. TAPLEY, D. F., J..biol. Chem.istry 222, 325 (1956). - 19. Novikorf, A. B., in: Subcellular partticles, Ronald Press Co., New York, 1959, 1-22. - 20. Mrshra, R. K., Rev. Canad. Biol. 19, 136 (1960).

Prof. Dr. H.-J. Merker 1000 Berlin 33

Königin-Luise-Str. 15 